\title{
A hybrid deep learning approach towards building an intelligent system for pneumonia detection in chest X-ray images
}

\author{
Ihssan S. Masad ${ }^{1}$, Amin Alqudah $^{2}$, Ali Mohammad Alqudah ${ }^{3}$, Sami Almashaqbeh $^{4}$ \\ 1,3,4 Department of Biomedical Systems and Informatics Engineering, Yarmouk University, Irbid, Jordan \\ ${ }^{2}$ Department of Computer Engineering, Yarmouk University, Irbid, Jordan
}

\begin{tabular}{l} 
Article Info \\
\hline Article history: \\
Received Sep 27, 2020 \\
Revised Jun 7, 2021 \\
Accepted Jun 17, 2021 \\
\hline
\end{tabular}

Keywords:

Convolutional neural network classification

Deep learning

\begin{abstract}
Pneumonia is a major cause for the death of children. In order to overcome the subjectivity and time consumption of the traditional detection of pneumonia from chest X-ray images; this work hypothesized that a hybrid deep learning system that consists of a convolutional neural network (CNN) model with another type of classifiers will improve the performance of the detection system. Three types of classifiers (support vector machine (SVM), k-nearest neighbor $(\mathrm{KNN})$, and random forest $(\mathrm{RF})$ were used along with the traditional CNN classification system (Softmax) to automatically detect pneumonia from chest X-ray images. The performance of the hybrid systems was comparable to that of the traditional CNN model with Softmax in terms of accuracy, precision, and specificity; except for the RF hybrid system which had less performance than the others. On the other hand, KNN hybrid system had the best consumption time, followed by the SVM, Softmax, and lastly the RF system. However, this improvement in consumption time (up to 4 folds) was in the expense of the sensitivity. A new hybrid artificial intelligence methodology for pneumonia detection has been implemented using small-sized chest X-ray images. The novel system achieved a very efficient performance with a short classification consumption time.
\end{abstract}

This is an open access article under the CC BY-SA license.

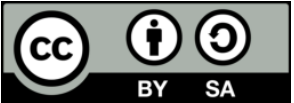

\section{Corresponding Author:}

Ihssan S. Masad

Department of Biomedical Systems and Informatics Engineering

Hijjawi Faculty for Engineering Technology

Yarmouk University

566 Shafiq Irshidat Street, Irbid 21163, Jordan

Email: imasad@yu.edu.jo

\section{INTRODUCTION}

Pneumonia is a respiratory condition in which lungs are affected by infection [1]. As the leading cause for the death of children under the age of 5, where it accounts for around $16 \%$ of all deaths of children [2]. Pneumonia kills over 800,000 children around the world every year [3], [4]. Adults can be affected by pneumonia as well, where over 50,000 people die every year, and more than one million people in the US (for example) are admitted to hospitals because of pneumonia; making it the most common cause of hospital admissions other than women giving birth [5]. Although pneumonia can be diagnosed by different imaging modalities such as magnetic resonance imaging (MRI) [6], [7] and computed tomography (CT) [8], [9], chest $\mathrm{X}$-ray imaging is still the most common method for pneumonia diagnosis, because it is cheap, fast, and clinically more available. 
Recent researches on diagnosis of pneumonia have focused on utilizing and developing automated computer-aided diagnosis (CAD) algorithms and methods to overcome the limitations of traditional (manual) diagnosis methods such as subjectivity, low accuracy, and time-consuming problems. Convolutional neural network $(\mathrm{CNN})$ is one of the most common used deep learning architectures that has been applied for classification of many medical images because of its efficiency in extracting different level useful features [10]-[14]. Gu et al. [15], for example, were able to distinguish pediatric bacterial from viral pneumonia through chest radiography images using deep convolutional neural network (DCNN) model. The distinction between these two types of pneumonia using CAD methods was challenging because both types have similar and confusing features. They found that the accuracy, area under the curve (AUC), as well as the sensitivity were better when extracting DCNN features than when extracting the handcraft features; however, neither DCNN nor handcraft methods achieved satisfactory high results which was justified by the unbalance in their data and overfitting during the training process [15]. In addition, Abiyev [13] showed that for the classification of chest common pathologies that might be found in chest radiography, DCNN performed better, in terms of accuracy and minimum square error, than other conventional learning approaches such as backpropagation neural network with supervised learning and competitive neural network with unsupervised learning.

The use of CNN models has shown a high accuracy for classification of chest X-ray images to diagnose pneumonia. For instance, Omar and Babalik [16] introduced a CAD system based on CNN to detect pneumonia from chest X-ray images with an accuracy of $87.65 \%$, which was higher than the accuracy obtained using different types of algorithms such as CheXNet [17], SMO, C4.5, and others [18]. On the other hand, Saraiva et al. [19] were able to achieve 95.3\% average accuracy using k-fold cross validation compared to $92.8 \%$ achieved by Kermany et al. [20] who used CNN with a transfer learning technique which has been proven to be more efficient with limited data. CNN models have been found to perform better than multilayer perceptron (MLP) as well, where CNN achieved an accuracy of 94.4\% compared to 92.16\% for MLP [21]. In another recent study, Stephen et al. [22] constructed a CNN-based model that was trained from scratch to extract useful features from chest X-ray images and detect if pneumonia infection is present with training and validation accuracy of $95.31 \%$ and $93.73 \%$, respectively.

The aforementioned studies have proven that CNN can be a reliable CAD model to diagnose and classify pneumonia from chest X-ray images because of the high accuracy it has achieved; however, there is still some loss (error) associated with all methods in the literature. Therefore, a novel hybrid artificial intelligence system has been proposed by this study. The proposed system is unique and distinguished from other existing systems by the use of convolutional neural network (CNN) along with other four different classifiers support vector machine (SVM), k-nearest neighbor (KNN), and random forest (RF), and Softmax. The main objective of this study was to investigate and evaluate the performance of this hybrid system in detecting pneumonia from small size chest X-ray images, which is another novel aspect of the study, by deep features extraction using a $\mathrm{CNN}$ fully connected layer.

\section{RESEARCH METHOD}

The block diagram of the proposed methodology is shown in Figure 1, where it started with transferring a learning applied technique to the pretrained CNN model. This transfer model was trained on the chest X-ray dataset to be used for deep feature extraction; then these features were fed to four types of classifiers; namely, Softmax, support vector machine (SVM), k-nearest neighbor (KNN), and random forest (RF). Finally, the performance of each classifier was evaluated and compared to that of other classifiers.

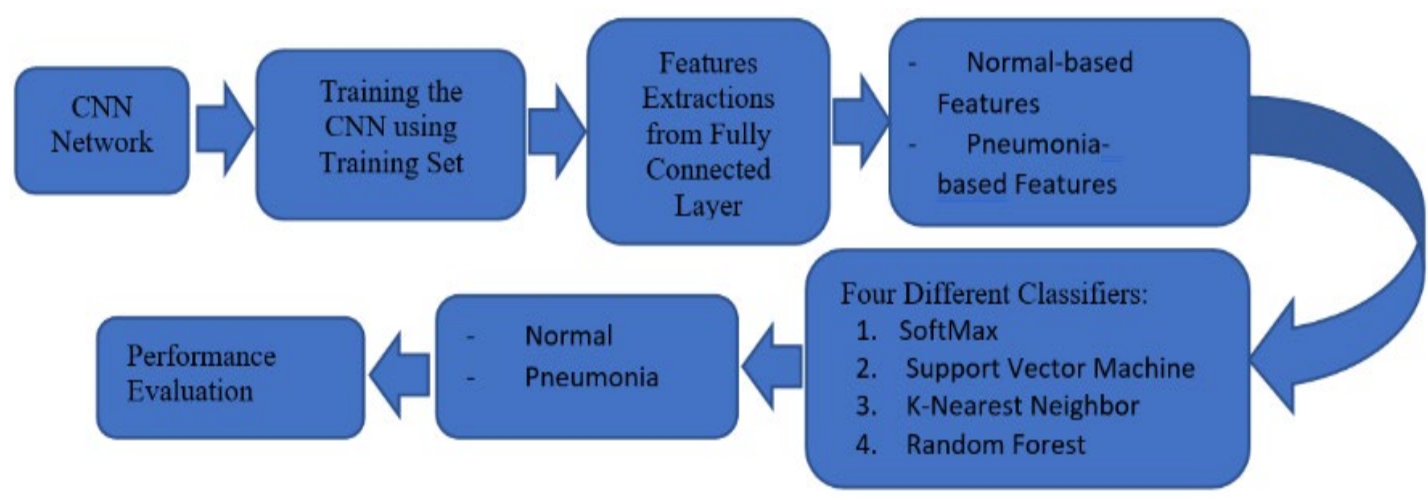

Figure 1. Block diagram of the proposed methodology 


\subsection{Dataset}

The chest X-ray images dataset used in this study as shown in Figure 2 was published online (https://data.mendeley.com/datasets/rscbjbr9sj/3) by kermany et al. [20]. The dataset was divided into three groups (training, testing, and validation) and inside each group, there were two subgroups (Pneumonia and Normal) chest X-ray images. The dataset contained 5,852 anterior-posterior chest X-ray images which were carefully chosen from retrospective pediatric patients with age group between 1 and 5 years. For dataset balance purposes, the original data groups were merged and categorized into two main groups (Normal and Pneumonia) then these two sets were rearranged into three subsets: training set, validation set, and testing set with portions of $70 \%, 15 \%$ and $15 \%$, respectively as illustrated in Table 1 .

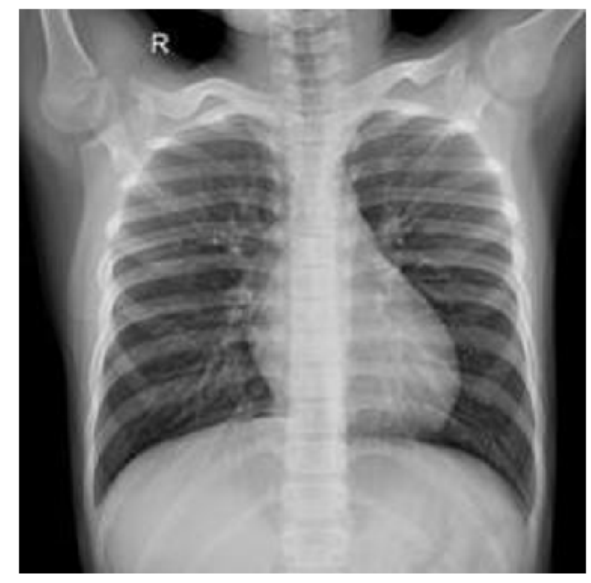

(a)

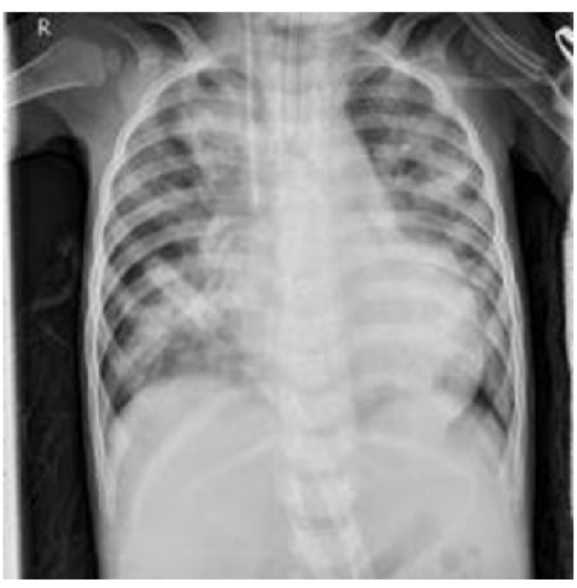

(b)

Figure 2. This figure are; (a) chest X-ray images from the used dataset for a normal lungc, (b) an infected lung with pneumonia

Table 1. The distribution of images used in the system

\begin{tabular}{ccccc}
\hline Case & Number of training images & Number of validation images & Number of testing images & Total number of images \\
\hline Normal & 1,107 & 237 & 237 & 1,581 \\
Pneumonia & 2,990 & 640 & 641 & 4,271 \\
Total & 4,097 & 877 & 878 & 5,852 \\
\hline
\end{tabular}

\subsection{CNN architecture}

This study has used a reconfigured CNN model that has been firstly proposed by Alqudah [23]. The model modification process included changing the input image layer size from $256 \times 256$ to $64 \times 64$ and the fully connected (FC) layer classes to 2 instead of 5. The modified CNN model layers details are shown in Table 2 while the architecture is shown in Figure 3. Like any CNN model, the proposed CNN model layers have been grouped into the following groups namely, deep features extraction layers and classification layers. For the deep feature extraction layers, each layer will take its input from the output of the preceding layer, then, the output of this layer will be processed as an input to the next layer; meanwhile, for the classification layers, this group of layers will take the features vector extracted using the previous group as an input received from the fully connected (FC) layer, usually, these layers are located at the end of all CNN model [13], [24].

\subsection{Deep feature extraction using $\mathrm{CNN}$}

The modified CNN model used in this study has been applied to the chest X-ray images dataset using transfer learning technique, after which, the trained model has been used for deep feature extraction from each X-ray image. The fully connected (FC) layer has been used for deep feature extraction. The FC layer is the layer that precedes the classification layer Softmax so its output will be a feature vector that contains the features as columns where each column represents one type of the classes [25]. This type of feature extraction methodology is completely automated and produces a very deep and representative features for the entire dataset especially when the used CNN model is well designed and a large dataset used [26]. In this method the dimension of the extracted features is $M x N$ where $M$ represents the number of entered data (Images) and $N$ is the number of classes [25]. 
Table 2. Layers specifications for the modified CNN architecture

\begin{tabular}{cccc}
\hline Layer \# & Layer name & \multicolumn{2}{c}{ Layer details } \\
\hline 1 & Input layer & Size & $64 \times 64$ \\
2 & Conv_1 & Number of filters & 48 \\
& & Kernel size & $3 \times 3$ \\
$3,7,11,15,18$ & Batch normalization & Number of channels & 32 \\
$5,9,13,20$ & Max pool & Kernel size & $2 \times 2$ \\
& & Stride & $2 \times 2$ \\
6 & Conv_2 & Number of filters & 32 \\
& & Kernel size & $3 \times 3$ \\
$4,8,12,16,19$ & RELU layer & Kernel size & $2 \times 2$ \\
& & Stride & $2 \times 2$ \\
10 & Conv_3 & Number of filters & 16 \\
& & Kernel size & $3 \times 3$ \\
& & Activation & RELU \\
14 & Conv_4 & Number of filters & 32 \\
& & Kernel size & $3 \times 3$ \\
17 & Conv_5 & Number of filters & 32 \\
& & Kernel size & $3 \times 3$ \\
\hline
\end{tabular}

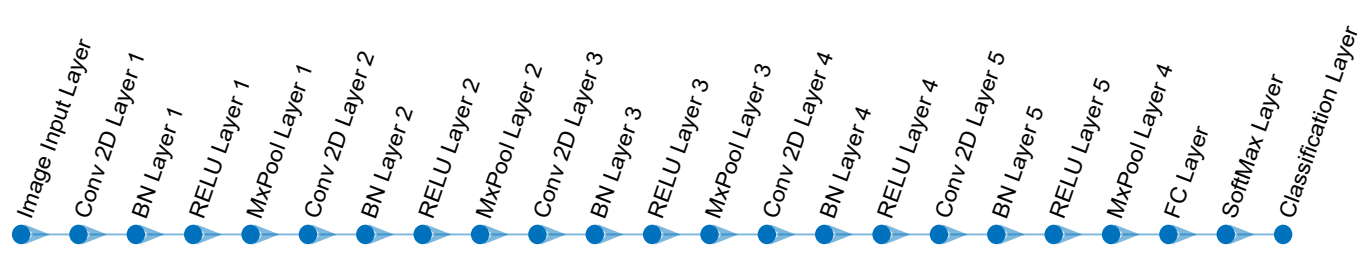

Figure 3. The architecture of the used CNN model

\subsection{Classification stage}

After the features have been extracted, different types of classifiers will be needed and used to find the corresponding label for every test image. For this purpose, four different types of classifiers have been used including SoftMax, SVM, KNN, and RF.

\subsubsection{Softmax classifier}

Softmax classifier is the most efficient and the simplest type of discriminatory classifier, which is used in CNN as the default classifier in the classification layer. The softmax discriminant classifier (SDC) finds the class of new features input of testing image by employing a nonlinear transformation function for the distance between the testing sample and training samples. In such a way, the learning method for the binary data in a softmax classifier is similar to the rule of standard binary data. The main difference between them is that softmax function is a generalization of the logistic sigmoid function, and it can deal with multiclass classification problems [25], [26].

\subsubsection{Support vector machine (SVM) classifier}

The support vector machine (SVM) classifier is a leading supervised machine learning algorithm that is widely used in medical applications to classify entire features into two classes. SVM builds the hyperplane model using training data that separates the entered data and can be used to anticipate the new feature class. The main aim of the SVM is mainly to find an optimal hyperplane that perfectly separates the entire data, and that maximizes the margin between the nearest data point called supporting points and the separating hyperplane [27], [28].

\subsubsection{K-nearest neighbor (KNN) classifier}

$\mathrm{K}$-nearest neighbor (KNN) algorithm is an instant-based, unsupervised, and non-parametric machine learning algorithm, which is very simple, lazy, and widely used for classification of medical data. In general, usually, the KNN receives an input data that contains the feature space and the target label; the output class of the input features space will be determined based on the majority voting technique of the neighbor's classes. The majority voting is applied on the weights which represent the distance between each feature point and the center of mass of the vector [28].

A hybrid deep learning approach towards building an intelligent system for pneumonia... (Ihssan S. Masad) 


\subsubsection{Random forest (RF) classifier}

Random forests (RF) classifier is an decision forests based ensemble classifier that has been proposed by Breiman [29] that is popular and widely used in multiclass medical data. The basic idea of RF methodology is to build classification trees by randomly selecting features from randomly selected samples with bagging strategy. Then, these trees are used to vote for a given input vector to get a class label. RF has many pros like efficiency on large-scale data, high precision, easy application, and overpowers in multi-class inputs, and it does not overfit.

\subsection{Performance evaluation}

In general, to make an evaluation and measure the performance of machine learning techniques used in this study, four well known statistical indices, namely, true positive (TP), false positive (FP), false negative (FN) and, true negative (TN) have been calculated. Then using these indices, the accuracy, precision, sensitivity, and specificity have been calculated according to (1)-(4) [30] shown:

$$
\begin{aligned}
& \text { Accuracy }=\frac{\mathrm{TP}+\mathrm{TN}}{\mathrm{TP}+\mathrm{FP}+\mathrm{TN}+\mathrm{FN}} \\
& \text { Sensitivity }=\frac{\mathrm{TP}}{\mathrm{TP}+\mathrm{FN}} \\
& \text { Specificity }=\frac{\mathrm{TN}}{\mathrm{FP}+\mathrm{TN}} \\
& \text { Precision }=\frac{\mathrm{TP}}{\mathrm{FP}+\mathrm{TP}}
\end{aligned}
$$

\section{RESULTS}

The methodology has been run using a desktop computer with Intel Core I7-6700 at 3.4 GHz and 16 GB of RAM and the code has been executed using a parallel environment. After feeding it with the training set, the CNN architecture has been trained using adaptive moment learning rate (ADAM) solver (initial learning rate of $10^{-3}$, mini batch size of 128 , and momentum of 0.9 ) in order to calculate the layers' weights; while the validation set was used for hyperparameters optimization. The training accuracy and loss for the used $\mathrm{CNN}$ architecture were plotted in Figure 4 which shows that after 620 iteration, the training accuracy achieved $100 \%$ while the loss reached $0 \%$; which means that the model has been trained efficiently on the training dataset.
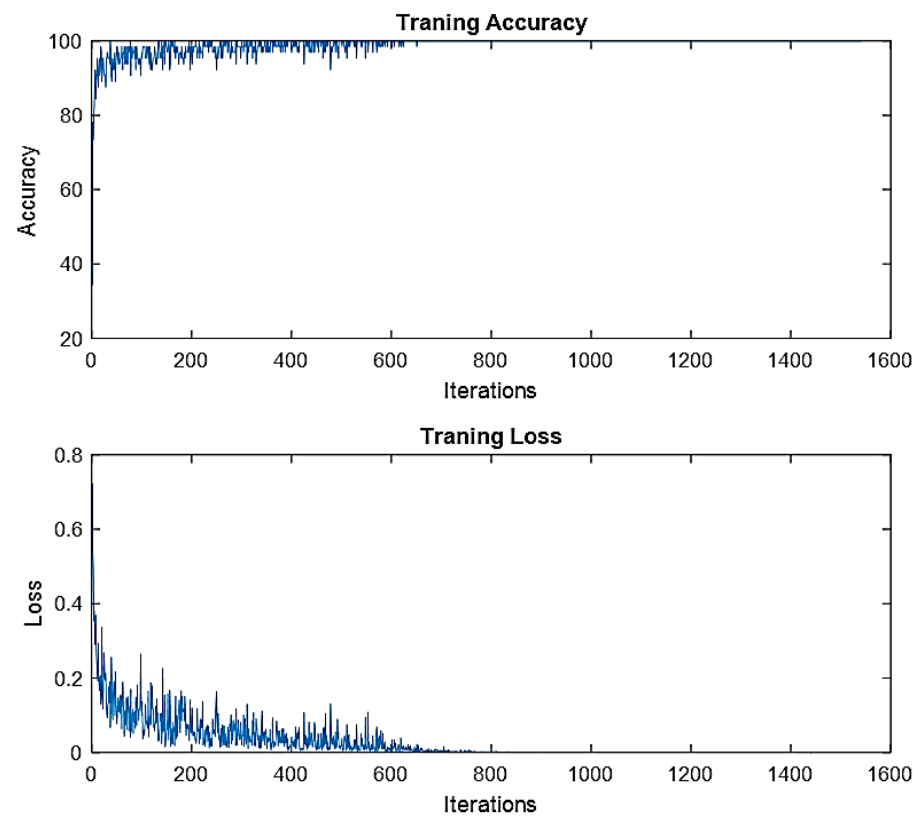

Figure 4. The training accuracy (top) and loss (bottom) change over the iterations 
After training the $\mathrm{CNN}$, the trained $\mathrm{CNN}$ has been used as a feature extractor from the fully connected (FC) layer. The output of this layer is a matrix with two columns representing the extracted deep features and rows representing the number of images for both training and testing datasets. Figure 5 shows the extracted deep features for both training and testing datasets.

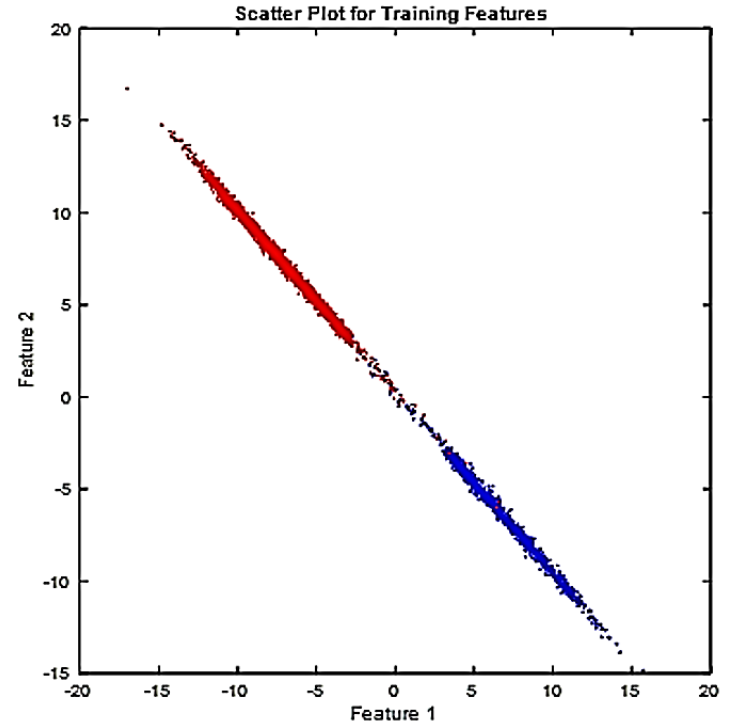

(a)

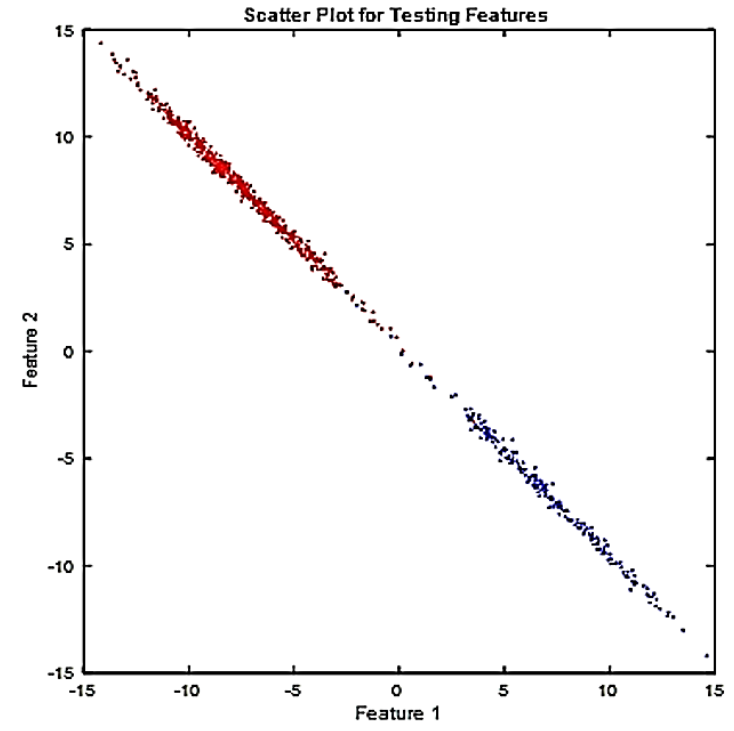

(b)

Figure 5. This figure are; (a) the extracted deep features for both training; and (b) testing dataset

To evaluate if the extracted deep features were significant, discriminant, and representative in the detection of the pneumonia, and to make a statistical check that extracted features were useful in detection pneumonia, a boxplot of each extracted deep feature among both classes was performed. As can be noticed from Figure 6, the range of holder exponents in the first feature and the second feature were far away from each other; which means that the extracted features can be used successfully in the detection of pneumonia. Also, it can be concluded from Figure 6 that the first feature was representative of the normal class (First Class), and the second feature was representative of the pneumonia class (Second Class).
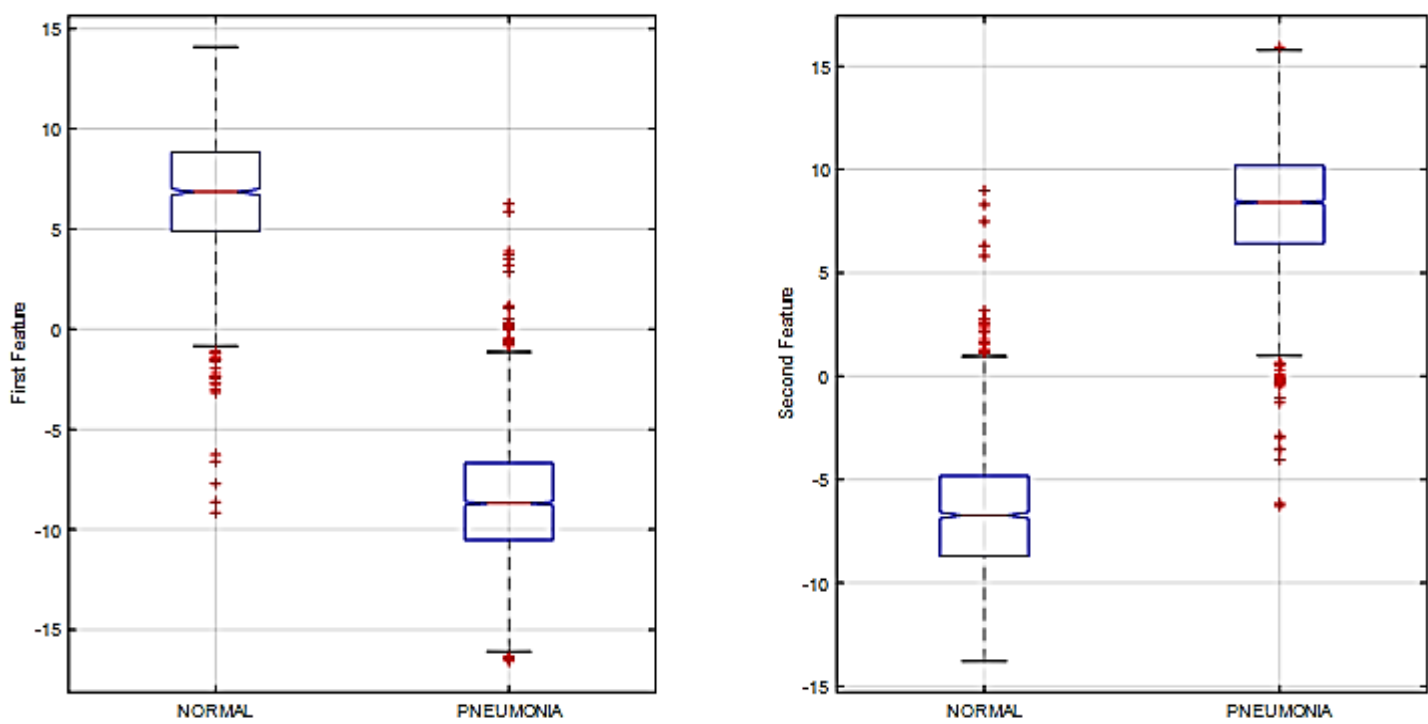

Figure 6. Boxplot for the both extracted features among the two classes 
The extracted features from the training and testing images were then fed to four different classifiers (SVM, KNN, RF, and Softmax). The KNN classifier was implemented with a number of neighbors value of 2 and chebyshev distance measurement, while SVM was implemented with a 9th order polynomial as a kernel function. Finally, the RF was implemented with a 20 bags to be used for Bootstrapping. The choice of classifiers parameters was made by testing different combinations of classifiers parameters on the training and testing datasets, then the parameters that led to the best performance have been selected [31], [32]. To check whether the selected parameters can be generalized, the classifiers models were evaluated using $10 \mathrm{~K}$ fold methodology. Figure 7 shows the confusion matrices for all classifiers, while Table 3 shows the training, testing, and overall accuracy of all classifiers. It can be noticed from Figure 7 and Table 3 that all classifiers performed very well in terms of overall accuracy of pneumonia detection with a small superiority of KNN.

Table 3. The accuracy of the four used classifiers

\begin{tabular}{cccc}
\hline Method & Training accuracy $\%$ & Testing accuracy $\%$ & Overall accuracy $\%$ \\
\hline Softmax & 99 & 99 & 99 \\
KNN & 100 & 98.5 & 99.3 \\
SVM & 99 & 99 & 99 \\
RF & 100 & 97.15 & 98.6 \\
\hline
\end{tabular}

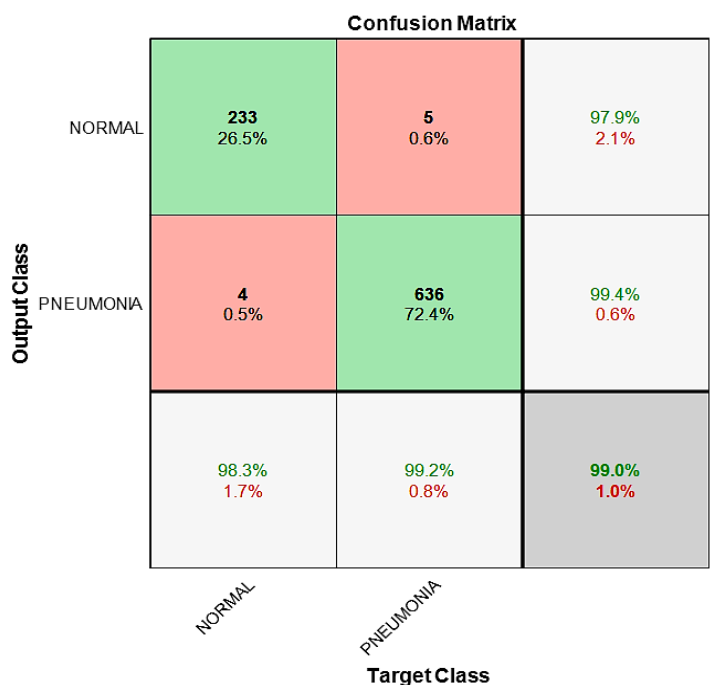

(a)

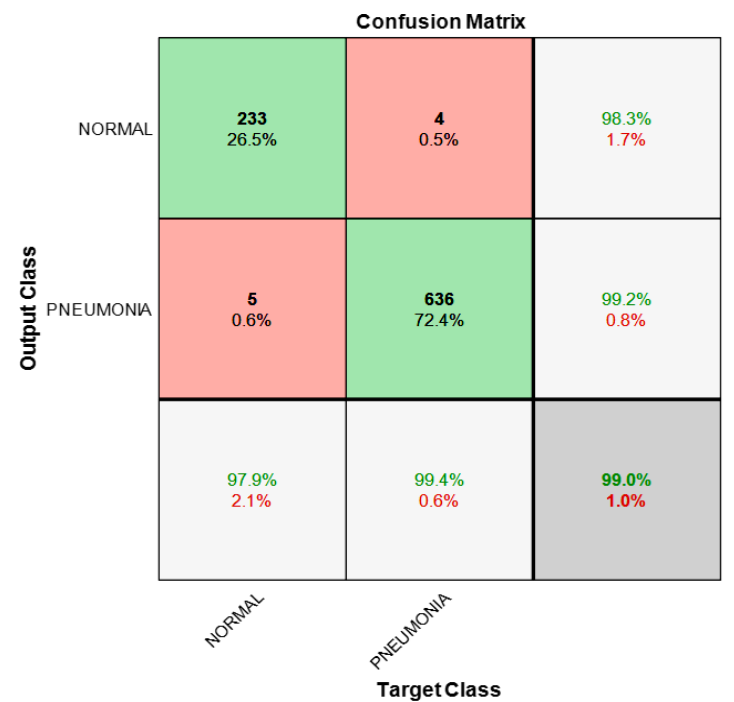

(c)

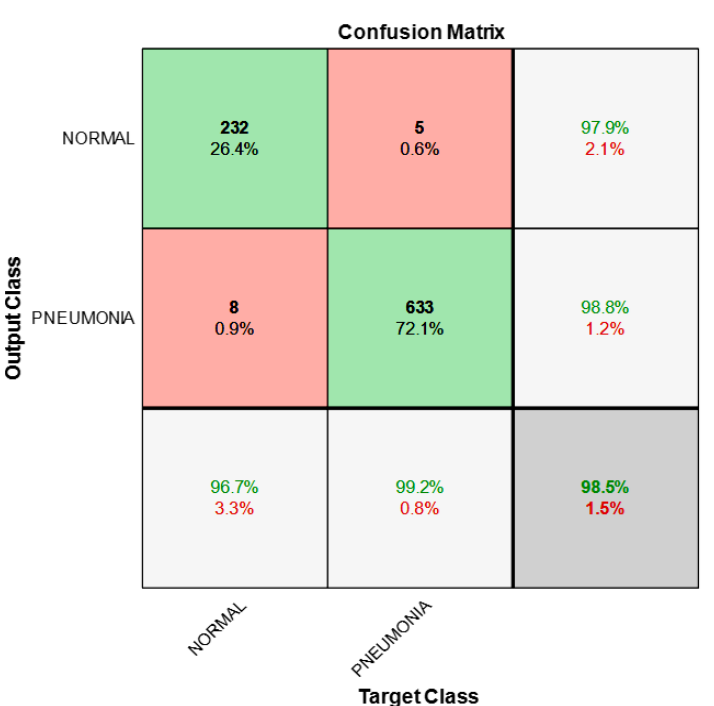

(b)

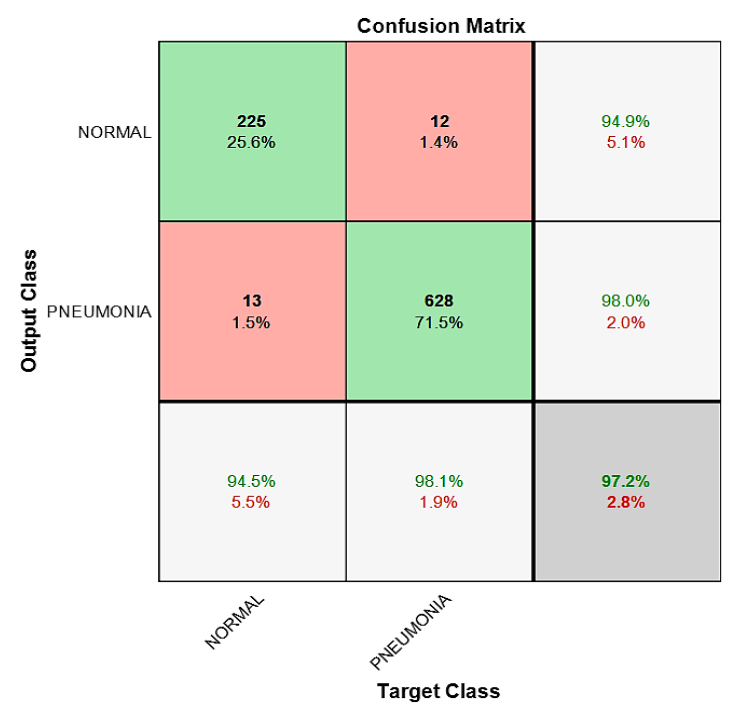

(d)

Figure 7. The testing confusion matrices for the used classifiers; (a) softmax; (b) KNN; (c) SVM; (d) RF 
In order to make a comprehensive comparison between classifiers performances, the performance metrics of these classifiers including accuracy, sensitivity, specificity, and precision have been calculated, and the average time for classifying the image for both classes has been measured as well. Figure 8 shows that the statistical performance of the Softmax classifier was $98.97 \%, 99.22 \%, 98.31 \%$, and $97.9 \%$, for accuracy, specificity, sensitivity and precision, respectively. SVM classifier achieved the same accuracy, specificity, and precision as Softmax but with a lower sensitivity of $97.9 \%$. KNN classifier, on the other hand, achieved a little lower performance evaluation with an accuracy of $98.51 \%$, a sensitivity of $96.67 \%$ and a precision of $97.89 \%$. Finally, RF classifier achieved the lowest performance with an accuracy of $97.15 \%$, and a sensitivity of $94.56 \%$, while the precision and specificity values were $94.96 \%$ and $98.12 \%$, respectively.

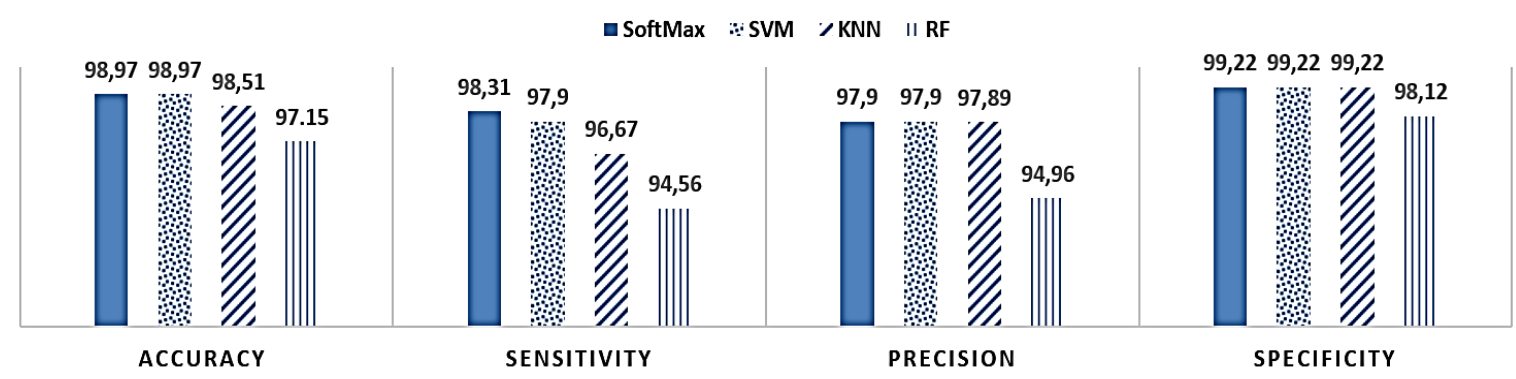

Figure 8. Performance comparison between different types of classifiers

Another evaluation of the system performance and stability using a $10 \mathrm{~K}$-fold methodology was applied to all used classifiers. The results showed that all classifiers performed as expected. Table 4 shows the performance of the proposed hybrid artificial system for pneumonia detection using a $10 \mathrm{~K}$-fold methodology. Figure 9 shows the time consumption for image classification using the different types of classifiers. The fastest classification was achieved by using KNN classifier, followed by SVM and Softmax; while the longest classification time was using the RF classifier.

Table 4. The accuracy of the four used classifiers using $10 \mathrm{~K}$-fold methodology

\begin{tabular}{ccccc}
\hline \multirow{2}{*}{ Measures } & \multicolumn{4}{c}{ Method } \\
& Softmax & KNN & SVM & RF \\
\hline Accuracy \% & $99.72 \pm 0.234$ & $98.61 \pm 0.117$ & $99.61 \pm 0.117$ & $97.61 \pm 0.234$ \\
Sensitivity \% & $99.52 \pm 0.325$ & $98.71 \pm 0.406$ & $99.71 \pm 0.212$ & $97.71 \pm 0.523$ \\
Precision \% & $98.45 \pm 0.483$ & $96.24 \pm 0.592$ & $97.24 \pm 0.592$ & $95.24 \pm 0.752$ \\
Specificity \% & $99.46 \pm 0.254$ & $98.58 \pm 0.234$ & $98.7 \pm 0.234$ & $97.58 \pm 0.534$ \\
\hline
\end{tabular}

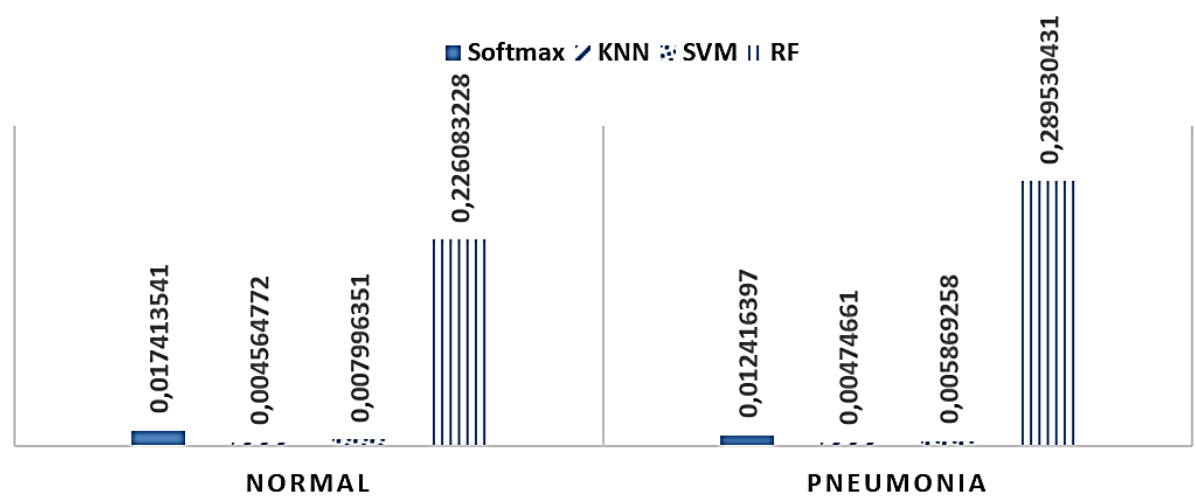

Figure 9. Classification time consumption for different types of classifiers

\section{DISCUSSION}

The goal of the current study was to investigate the impact of employing a hybrid artificial intelligence system on the performance of pneumonia diagnosis using chest X-ray images; as well as to

A hybrid deep learning approach towards building an intelligent system for pneumonia ... (Ihssan S. Masad) 
examine the effect of using a new CNN architecture (AOCT-NET) with small image input sizes $(64 \times 64)$ on the efficiency of pneumonia detection. In the training phase scheme, all of the used classifiers (KNN, SVM, Softmax, and RF) achieved high accuracy using two extracted features; while during the testing phase, the four classifiers varied in their performance.

When comparing the experimental results of the proposed hybrid system to previous methods in the literature, it can be noticed that deep features extraction methods scored an accuracy less than that of the proposed methods (>97\%). Rajpurkar et al. [17], for instance, proposed a CNN (ChexNet) that extracted deep features and used the Softmax classifier with an accuracy of $76.80 \%$; while Saul et al. achieved a little higher accuracy with a value of 78.73\% [24]. Both Rajpurkar et al. [17] and Saul et al. [24] used the NIH Chest X-ray 14 image dataset which contained more than 122,000 chest X-ray images of 15 different classes (normal and 14 diseases) among which 325 for pneumonia detection. The authors of the dataset have distributed the dataset into training, validation, and testing files. On the other hand, Kermany et al. [20] achieved a significant improvement in the accuracy $(92.8 \%)$ when they used a transfer learning technique applied on Inception V3 CNN with Softmax classifier. More recently, Saraiva et al. [21] achieved an accuracy of $92.16 \%$ when they used deep features extracted using three hidden layers of MLP structure and Softmax classifier and an accuracy of $94.40 \%$ using deep features extracted using CNN architecture of three layers with Softmax classifier; both methods used the Kermany's X-ray pneumonia dataset that consisted of 5,852 images separated into training, validation, and testing files; they used image input size of $150 \times 150$ pixels. Also, Saraiva et al. [19] reported reaching an accuracy of $95.30 \%$ when using deep features with a Softmax classifier using ten layers $\mathrm{CNN}$ and input size of $300 \times 300$ pixels. A summary of the comparison between the proposed methodology and other methods in the literature is listed in Table 5.

Table 5. Comparison between the accuracy results in the proposed method and in other methods

\begin{tabular}{|c|c|c|c|}
\hline Reference & Features Set & Classifier & Accuracy $(\%)$ \\
\hline Rajpurkar et al. [17] & Deep features & Softmax & 76.80 \\
\hline Saraiva et al. [19] & Deep features & Softmax & 95.30 \\
\hline Kermany et al. [20] & Deep features using transferee learning of inception V3 CNN & Softmax & 92.80 \\
\hline Saraiva et al. [21] & Deep features using multilayer perceptron (MLP) & Softmax & 92.16 \\
\hline Saraiva et al. [21] & Deep features & Softmax & 94.40 \\
\hline Saul et al. [24] & Deep features using nine layered ResNet CNN applied on increased contrast & Softmax & 78.73 \\
\hline \multirow[t]{4}{*}{ Current Study } & & Softmax & 99.00 \\
\hline & \multirow{3}{*}{ Deep features } & KNN & 98.50 \\
\hline & & SVM & 99.00 \\
\hline & & RF & 97.15 \\
\hline
\end{tabular}

The results of this study showed that Softmax and SVM achieved the highest performance in terms of accuracy, sensitivity, specificity, and precision, which can be explained by the fact that this study had only two classes (Normal and Pneumonia) and these two classifiers are well known to outperform other classifiers in binary problems because the Softmax classifier is a generalized version of binary logistic regression classifier, where SVM is known to be a generalized classifier for binary problems that use hyperplane to separate features into two classes. However, RF and $\mathrm{KNN}$ are more commonly used in multiclass classification $(\mathrm{RF})$ and clustering $(\mathrm{KNN})$ problems.

On the other hand, comparing the consumption time for testing image classification using the four classifiers, the fastest classifier for both: normal and pneumonic cases was the KNN, then SVM, Softmax, and lastly the RF. As mentioned before, $\mathrm{KNN}$ is a clustering technique which requires less time compared to other methods for making decision [33]; also, SVM is a hyperplane separation method which is a fast classification method [33]. Combining both characteristics of the system (i.e., the performance and consumption time), it was noticed that the consumption time can be reduced by a factor of 4 when using the KNN classifier compared to Softmax classifier, however, this decrease was traded off by sacrificing some sensitivity and accuracy. Similarly, the use of SVM classifier decreased the consumption time to half compared to Softmax classifier in the expense of sensitivity.

\section{CONCLUSION}

In conclusion, a novel hybrid artificial intelligence methodology for pneumonia detection has been implemented using small-sized chest X-ray images. The hybrid artificial intelligence system was built using a CNN model that was pretrained on OCT images. In contrary to other studies in the literature that utilized the transfer learning approach using pre-trained CNN architecture only, the hybrid system used in this work utilized the pre-trained $\mathrm{CNN}$ architecture for features extraction, and another classifier for making the 
decision. The hybrid system achieved a very high performance especially in terms of accuracy with short classification consumption time that varied with the classifier type.

\section{REFERENCES}

[1] National Institutes of Health, "NLM Community-acquired pneumonia in adults," MedlinePlus. Accessed on Feb 09 2020. [Online]. Available: https://medlineplus.gov/ency/article/000145.htm

[2] ATC, "Top 20 Pneumonia Facts. American Thoracic Society," 2018. [Online]. Available: https://www.thoracic.org/patients/patient-resources/resources/top-pneumonia-facts.pdf

[3] WHO, "Pneumonia Facts Sheet," World Health Organization," 2017. [Online]. Available: https://www.who.int/news-room/fact-sheets/detail/pneumonia

[4] UNICEF, "Childhood pneumonia: Everything you need to know," United Nations International Children's Emergency Fund, 2020. [Online]. Available: https://www.unicef.org/stories/childhood-pneumonia-explained

[5] CDC, "Pneumonia," Centers for Disease Control and Prevention, 2020. [Online]. Available: https://www.cdc.gov/pneumonia/prevention.html.

[6] A. Yikilmaz, A. Koc, A. Coskun, K. M. Ozturk, V. R. Mulkern and Y. E. Lee, "Evaluation of pneumonia in children: comparison of MRI with fast imaging sequences at $1.5 \mathrm{~T}$ with chest radiographs," Acta Radiologica, vol. 52, no. 8, pp. 914-919, 2011, doi: 10.1258/ar.2011.100429.

[7] I. U. Attenberger et al., "3 Tesla proton MRI for the diagnosis of pneumonia/lung infiltrates in neutropenic patients with acute myeloid leukemia: initial results in comparison to HRCT," European journal of radiology, vol. 83, no. 1, pp. e61-e66, 2014, doi: 10.1016/j.ejrad.2013.09.002.

[8] Y. Claessens et al., "Early Chest Computed Tomography Scan to Assist Diagnosis and Guide Treatment Decision for Suspected Community-acquired Pneumonia," American Journal of Respiratory and Critical Care Medicine, vol. 192, no. 8, pp. 974-982, 2015, doi: 10.1164/rccm.201501-0017OC.

[9] V. Prendki et al., "Low-dose computed tomography for the diagnosis of pneumonia in elderly patients: a prospective, interventional cohort study," European Respiratory Journal, no. 51, no. 5, 2018, Art. no. 1702375, doi: 10.1183/13993003.02375-2017.

[10] E. G. Hinton, S. Osindero, and W. Y. The, "A fast learning algorithm for deep belief nets," Neural Computation, vol. 18, no. 7, pp. 1527-1554, 2006, doi: 10.1162/neco.2006.18.7.1527.

[11] M. Avendi, A. Kheradvar and H. Jafarkhani, "A combined deep-learning and deformable-model approach to fully automatic segmentation of the left ventricle in cardiac MRI," Medical Image Analysis, no. 30, pp. 108-119, 2016, doi: 10.1016/j.media.2016.01.005.

[12] C. H. Shin, K. Roberts, L. Lu, D. Demner-Fushman, J. Yao, and M. R. Summers, "Learning to read chest x-rays: Recurrent neural cascade model for automated image annotation," Proceedings of the IEEE conference on computer vision and pattern recognition, 2016, pp. 2497-2506, doi: 10.1109/CVPR.2016.274.

[13] R. Abiyev, and K. M. Ma'aitah, "Deep Convolutional Neural Networks for Chest Diseases Detection," Journal of Healthcare Engineering, vol. 2018, 2018, Art. no. 4168538, doi: 10.1155/2018/4168538.

[14] P. Lakhani, and B. Sundaram, "Deep learning at chest radiography: automated classification of pulmonary tuberculosis by using convolutional neural networks," Radiology, vol. 284, no. 2, pp. 574-582, 2017, doi: 10.1148/radiol.2017162326.

[15] X. Gu, L. Pan, H. Liang, and R. Yang, "Classification of bacterial and viral childhood pneumonia using deep learning in chest radiography," Proceedings of the 3rd International Conference on Multimedia and Image Processing, 2018, pp. 88-93, doi: 10.1145/3195588.3195597.

[16] S. H. Omar, and A. Babalık, "Detection of Pneumonia from X-Ray Images using Convolutional Neural Network," Proceedings Book, 2019, pp. 183-185.

[17] P. Rajpurkar et al., "Chexnet: Radiologist-level pneumonia detection on chest x-rays with deep learning," arXiv preprint arXiv:1711.05225, 2017.

[18] E. I. Livieris, A. Kanavos, V. Tampakas, and P. Pintelas, "A Weighted Voting Ensemble Self-Labeled Algorithm for the Detection of Lung Abnormalities from X-Rays," Algorithms, vol. 12, no. 3, pp. 1-15, 2019, doi: 10.3390/a12030064.

[19] A. A. Saraiva et al., "Classification of Images of Childhood Pneumonia using Convolutional Neural Networks," BIOIMAGING, pp. 112-119, 2019, doi: 10.5220/0007404301120119.

[20] D. Kermany et al., "Identifying medical diagnoses and treatable diseases by image-based deep learning," Cell, vol. 172, no. 5, pp. 1122-1131, 2018, doi: 10.1016/j.cell.2018.02.010.

[21] A. A. Saraiva et al., "Models of Learning to Classify X-ray Images for the Detection of Pneumonia using Neural Networks," Bioimaging, pp. 76-83, 2019, doi: 10.5220/0007346600760083.

[22] O. Stephen, M. Sain, J. U. Maduh, and U. D. Jeong, "An efficient deep learning approach to pneumonia classification in healthcare," Journal of healthcare engineering, vol. 2019, 2019. Art. no. 4180949, doi: 10.1155/2019/4180949.

[23] A. M. Alqudah, "AOCT-NET: a convolutional network automated classification of multiclass retinal diseases using spectral-domain optical coherence tomography images," Medical \& biological engineering \& computing, vol. 58, no. 1, pp. 41-53, 2020, doi: 10.1007/s11517-019-02066-y.

[24] J. C. Saul, Y. D. Urey, and D. C. Taktakoglu, "Early diagnosis of pneumonia with deep learning," arXiv preprint arXiv:1904.00937, 2019.

[25] J. D. Trosten and P. Sharma, "Unsupervised feature extraction-a cnn-based approach," Scandinavian Conference on Image Analysis, vol. 11482, 2019, pp. 197-208, 10.1007/978-3-030-20205-7_17.

A hybrid deep learning approach towards building an intelligent system for pneumonia... (Ihssan S. Masad) 
[26] D. Garcia-Gasulla, "On the behavior of convolutional nets for feature extraction," Journal of Artificial Intelligence Research, vol. 61, no. 1, pp. 563-592, 2018, doi: 10.1613/jair.5756.

[27] A. M. Alqudah, and M. A. Alqudah, "Sliding Window Based Support Vector Machine System for Classification of Breast Cancer Using Histopathological Microscopic Images," IETE Journal of Research, vol. 7, pp. 1-9, 2019, doi: 10.1080/03772063.2019.1583610.

[28] A. M. Alqudah, "Ovarian Cancer Classification Using Serum Proteomic Profiling and Wavelet Features A Comparison of Machine Learning and Features Selection Algorithms," Journal of Clinical Engineering, vol. 44, no. 4, pp. 165-173, 2019, doi: 10.1097/JCE.0000000000000359.

[29] M. A. Alqudah, A. Albadarneh, I. Abu-Qasmieh, and H. Alquran, "Developing of robust and high accurate ECG beat classification by combining Gaussian mixtures and wavelets features," Australasian physical \& engineering sciences in medicine, vol. 42, no. 1, pp. 149-157, 2019, doi: 10.1007/s13246-019-00722-z.

[30] A. Alqudah, H. Alquraan, I. Abu-Qasmieh, and A. Al-Badarneh, "Employing Image Processing Techniques and Artificial Intelligence for Automated Eye Diagnosis Using Digital Eye Fundus Images," Journal of Biomimetics, Biomaterials and Biomedical Engineering, vol. 39, pp. 40-56, 2018, doi: 10.4028/www.scientific.net/JBBBE.39.40.

[31] F. Albu and D. Martinez, "The application of support vector machines with Gaussian kernels for overcoming cochannel interference," Neural Networks for Signal Processing IX: Proceedings of the 1999 IEEE Signal Processing Society Workshop (Cat. No. 98TH8468), 1999, pp. 49-57, doi: 10.1109/NNSP.1999.788122.

[32] A. M. Alqudah, M. H. Algharib, M. A. Algharib and M. H. Algharib, "Computer aided diagnosis system for automatic two stages classification of breast mass in digital mammogram images," Biomedical Engineering: Applications, Basis and Communications, vol. 31, no. 1, 2019, Art. no. 1950007, doi: $10.4015 / \mathrm{S} 1016237219500078$.

[33] M. A. Anter and E. A. Hassenian, "Normalized multiple features fusion based on PCA and multiple classifiers voting in CT liver tumor recognition," Advances in Soft Computing and Machine Learning in Image Processing, vol. 730, 2018, pp. 113-129, doi: 10.1007/978-3-319-63754-9_6. 